\title{
TEOREMA DE APROXIMAÇÃO
}

\author{
Joabe dos Santos Machado'; Maurício de Araujo Ferreira² \\ 1. Bolsista PIBIC/FAPESB, Graduando em Licenciatura em Matemática, Universidade Estadual de Feira de Santana, \\ e-mail: joabe_machado@ hotmail.com \\ 2. Orientador, Departamento de Ciências Exatas, Universidade Estadual de Feira de Santana, e-mail: \\ maferreira@uefs.br
}

PALAVRAS-CHAVE: aproximação; valorização.

\section{INTRODUÇÃO}

Neste trabalho, buscando compreender seu funcionamento, o teorema de aproximação teve um papel central. De acordo com algumas fontes, esse teorema teve sua primeira aparição e demonstração no artigo de Artin-Whaples [1945]. E Hasse reproduziu a demonstração desse teorema na segunda edição de seu livro "Zahlentheorie". Na primeira edição, de 1938, Hasse prova o Teorema da Aproximação para o casoem que o corpo K éum corpo de números algébricos ou um corpo de funções algébricas sobre um corpo finito e para primos distintos $P_{1}, \ldots, P_{r}$. Posteriormente, foi generalizado para corpos que são extensões algébricas finitas $\mathrm{L}$ de $\mathrm{K}$, com relação a um número finito de primos de L, estendendo os primos $P_{i}$ de K. Neste trabalho estudamos o Teorema de Aproximação para valorizações independentes em corpos arbitrários.

\section{MATERIAL E MÉTODOS OU METODOLOGIA (ou equivalente)}

A pesquisa foi de natureza teórica. O ponto de partida foi o levantamento bibliográfico. Neste ponto, aprendemos os resultados que já eram conhecidos e as técnicas utilizadas para obtê-los. Foi estudado principalmente o Teoremas de Aproximação para valorizações em corpos.

\section{RESULTADOS E/OU DISCUSSÃO (ou Análise e discussão dos resultados)}

Para que o Teorema da Aproximação seja compreendido, é necessário que alguns outros conceitos e proposições sejam estudados. Assim, buscou-se, neste trabalho, a realização mais minuciosa de tais proposições. O que poderá facilitar estudos posteriores sobre tal tema. 
Definição 1.: Seja K um corpo e $\Gamma$ um grupo abeliano ordenado. Uma valorização $v$ de $K$ é uma aplicação $v: K \rightarrow \Gamma \cup\{\infty\}$ uma valorização que satisfaz as seguintes propriedades:

1. $v(x)=\infty \Leftrightarrow x=0$

2. $v(x+y) \geq \min \{v(x), v(y)\}$;

3. $v(x y)=v(x)+v(y)$.

Teorema 1.: Seja $v: K \rightarrow \Gamma \cup\{\infty\}$ uma valorização de K. Para cada $\gamma \in K$ e cada $a \in \Gamma$ definimos o conjunto

$$
U_{\gamma}(a):=\{x \in K: v(x-a)>\gamma\} .
$$

Estes conjuntos formam uma base aberta de vizinhanças em torno do ponto a.

Demonstração: (i) $a \in U_{\gamma}(a)$, pois, $v(a-a)=v(0)=\infty>\gamma$

(ii) $U_{\gamma}(a) \cap U_{\lambda}(a)=U_{\delta}, \operatorname{com} \delta=\max \{\gamma, \lambda\}$. De fato, $U_{\gamma}(a) \cap U_{\lambda}(a)=\{x \in v(x-a)>\gamma\} \cap\{x \in K: v(x-a)>\lambda\}=\{x \in K: v(x-a)>$ $\max \{\gamma, \lambda\}\}$

(iii) Se $b \in U_{\gamma}$ e $b \neq a$, temos que $v(b-a)=\gamma^{\prime}$. Logo, $U_{\gamma^{\prime}}(b) \subseteq U_{\gamma}(a)$.

Teorema 2.: Dois anéis de valorização de $\mathrm{K}$ não triviais $O_{1}$ e $O_{2}$ são dependentes se, e somente se, eles induzem a mesma topologia em K.

Demonstaração: Uma vez que dois anéis de valorizações dependentes têm um engrossamento não trivial comum, para mostrar que eles induzem a mesma topologia, basta considerar o caso particular $O_{1} \subseteq O_{2}$. De fato, por definição, duas valorizações são dependentes se $O_{1} O_{2} \subset K$ com $O_{1} O_{2} \neq K$. Como $O_{1} \subset O_{1} O_{2}$, temos que $O_{1}$ e $O_{1} O_{2}$ induzem a mesma topologia. Analogamnete, concluímos que $\mathrm{O}_{2} \subset \mathrm{O}_{1} \mathrm{O}_{2}$. Desse modo, conclúmos que $O_{1}$ e $O_{2}$ induzem a mesma topologia. Seja $v: K \rightarrow \Gamma \cup\{\infty\}$ uma valorização correspondente a $O_{1}$. Pelo Lema 1 e suas consequências, existe um subgrupo convexo $\Delta$ de $\Gamma$ associado a $O_{2}$ tal que $K^{\times} \longrightarrow \Gamma \longrightarrow \Gamma / \Delta=\Gamma_{2}$ é uma valorização $v_{2}$ associada a $\mathrm{O}_{2}$, sendo $\mathrm{O}_{2} \neq \mathrm{K}$ e $\Gamma \neq\{0\}$. Sendo

$$
\begin{gathered}
U_{\gamma}(0)=\{a \in K: v(a)>\gamma\} \\
U_{v+\Delta}(0)=\left\{a \in K: v_{2}(a)>v+\Delta\right\}=\{a \in K: v(a)>\lambda \forall \lambda \equiv \gamma \bmod \Delta\}
\end{gathered}
$$

Desse modo, conluímos que $U_{v+\Delta}(0) \subseteq U_{\gamma}(0)$

Por outro lado, se $v(a)>2 \gamma$, então $v_{2}(a) \geq 2 \gamma+\Delta$. Assim, se $\gamma>0$ e também $\left.v_{(} a\right) \leq \gamma+\Delta$, então $\gamma+\Delta \geq 2 \gamma+\Delta$. Consequentemente, $\gamma \in \Delta$. Logo, para $0<\gamma$ não pertecente a $\Delta$, segue que $U_{2 \gamma} \subseteq U_{v+\Delta}(0)$. 
Logo as topologias induzidas por $O_{1}$ e $O_{2}$ são idênticas. Concluímos, desse modo, que as topologias induzidas de $O_{1}$ e $O_{2}$ são idênticas. Consideremos, agora, $M_{1}$ e $M_{1}$ ideais maximais de $O_{1}$ e $O_{2}$, respectivamente. Se $M_{1}$ e $M_{1}$ induzem a mesma topologia em $K$, então $M_{2}$ é uma vizinhança aberta de 0 na topologia induzida por $O_{1}$. Consequentemente, existe existe um $a \in K_{x}$ com $a M_{1} \subseteq M_{2}$. Como $M_{2}$ é um ideal maximal do anel de valorização $\mathrm{O}_{2}, 0$ conjunto $K \backslash M_{2}$ é fechado para multiplicação. Assim podemos formar 0 anel

$$
O_{3}=\left\{\frac{x}{y}: x \in O_{1}, y \in O_{1} \backslash M_{2}\right\}
$$

Como $O_{3}$ contém $O_{1}$, este também é uma anel de valorização. $O_{3}$ também contém $O_{2}$. De fato, se $x \in O_{2} \backslash\{0\}$, então $x^{-1}$ não pertence a $M_{2}$. Consequentemente, $x=1 / x^{-1} \in O_{3}$ para $x^{-1} \in O_{1}$. Se, entretanto, $x^{-1}$ não pertence a $O_{1}$, temos que $x \in O_{1} \mathrm{e}$, consequentemente, $x \in O_{3}$. Finalmente $O_{3} \neq K$. De fato, seja $z \in M_{1} \backslash\{0\}$. Então, $\frac{1}{a z}$ não pertence a $O_{3}$. Podemos ver isto fazendo $\frac{1}{a z}=\frac{x}{y}$, com $x \in O_{1}$ e $y \in O_{1} \backslash M_{2}$. Então $y=a(z x) \in a M_{1} \subseteq M_{2}$, uma contradição. Logo, provamos que $O_{1} \mathrm{e} O_{2}$ são dependentes.

Lema 1.: Seja $O$ um anel de valorização de $K$ não-trivial correspondente a valorização $v: K \rightarrow \Gamma \cup\{\infty\}$. Então existe uma correspondência 1-1 de subgrupos convexos $\Delta$ de $\Gamma$ com ideais primos p de $O$, e consequentemente com os anéis que contém $O$. Essa correpondência é dada por

$$
\begin{gathered}
\Delta \mapsto \mathbf{p}_{\Delta}=\{x \in K: v(x)>\delta \forall \delta \in \Delta\} \\
\mathbf{p} \mapsto \Delta_{\mathbf{p}}=\{\gamma \in \Gamma: \gamma,-\gamma<v(x) \forall x \in \mathbf{p}\} .
\end{gathered}
$$

Em particular, se $O$ tem classificação finita, essa classificação coincide com a dimensão de Krull de $O$.

Teorema 3(Teorema da Aproximação): Suponha que $O_{1}, \ldots, O_{n}$ sejam anéis de valorização independentes de $K$. Para cada $i$ tal que $1 \leq i \leq n$, seja $v_{i}: K \rightarrow \Gamma_{i} \cup\{\infty\}$ uma valorização correspondente a $O_{i}$. Então, para cada $a_{1}, \ldots, a_{n} \in K$ e $\gamma_{1} \in \Gamma_{1}, \ldots, \gamma_{n} \in \Gamma_{n}$, existe um $x \in K$ com $v_{i}\left(x-a_{i}\right)>\gamma_{i}$ para todo $i \in\{1, \ldots, n\}$

Demonstração: Para cada $i$ tal que $1 \leq i \leq n$ escolhemos $\delta_{i} \in \Gamma_{i}$, sastifazendo $\delta_{i} \geq \gamma_{i} \mathrm{e}$ $-\delta_{i} \leq v_{i}\left(a_{1}\right), \ldots, v_{i}\left(a_{n}\right)$. Algumas novas restições serão impostas para cada $\delta_{i}$ no decorrer desta demonstração.

Consideremos os conjuntos abertos $M_{i}=\left\{x \in K: 2 \delta_{i}<v_{i}(x)\right\}$ e $A_{i}=\left\{x \in K:-2 \delta_{i} \leq\right.$ $v(x)\}$.

(1) Podemos escolher o $\delta_{i}$ de tal forma que

$$
M_{1} \cap \bigcap_{j=2}^{n}\left(K \backslash A_{j}\right) \neq \emptyset
$$

Para provar (1), vamos usar indução em $n$. 
$n=2$ : se $M_{1} \cap\left(K \backslash A_{2}\right)=\emptyset$, então $M_{1} \subseteq A_{2}$. Escolhendo $c_{i} \in M_{i}(i=1,2)$, vemos que $c_{i} A_{i} \subset M_{i}$ e $c_{i} M_{i} \subset M_{i}$. Portanto, como $M_{1} \subset A_{2}$, temos que

$$
\left(c_{2} c_{1}\right) M_{1}=c_{2}\left(c_{1} M_{1}\right) \subseteq c_{2} M_{1} \subseteq c_{2} A_{2} \subseteq M_{2}
$$

Fazendo $a=c_{2} c_{1}$, vemos que $a M_{1} \subseteq M_{2}$, e recaímos exatamente na demonstração do Teorema 2. Assim, concluímos que $\mathrm{O}_{1}$ e $\mathrm{O}_{2}$ são dependentes, o que é uma contradição. Pela hipótese de indução, existe $r \in M_{1} \cap\left(K \backslash A_{2}\right)$. Escolhemos $\delta_{3}, \ldots, \delta_{n}$ sufucientemente grandes para que $e \in A_{j}$ para todo $j=3, \ldots, n$. Pela hipótese de indução, existe ainda um

$$
s \in M_{1} \cap \bigcap_{3 \leq j \leq n}\left(K \backslash A_{j}\right)
$$

Se $s \notin A_{2}$, a prova está feiota.No caso em que $s \in A_{2}$, como todo $M_{i}$ e todo $A_{i}$ é fechado na adição e na subtração,

$$
s+r \in M_{1} \cap \bigcap_{2 \leq j \leq n}\left(K \backslash A_{j}\right),
$$

provamos (1).

analogamente, encontramos, por meio de $\delta_{v}$ que $M_{1} \cap \bigcap_{j \neq i}\left(K \backslash A_{j}\right) \neq \emptyset$.

Um elemento desta intercessão se aproxima do infinito com respeito a $v_{j}$ para cada $j \neq i$, e ele se aproxima de 0 em relação a $v_{i}$.

(2) Segue-se agora que

$$
\left(1+M_{i}\right) \cap \bigcap_{j \neq i} M_{j} \neq \emptyset .
$$

De fato,

$$
x \in M_{i} \Rightarrow \frac{1}{1+x}=1-\frac{x}{1+x} \in 1+M_{i},
$$

e

$$
x \in K \backslash A_{j} \Rightarrow v_{j}(1+x)=v_{j}(x), \text { daí } \frac{1}{1+x} \in M_{j} .
$$

(2) Então escolhemos $d_{i} \in\left(1+M_{i}\right) \cap \bigcap_{j \neq i} M_{j}$ e, finalmente, definimos o conjunto

$$
x:=a_{1} d_{1}+\ldots+a_{n} d_{n} .
$$

Como $d_{i}-1 \in M_{i} \forall j \neq i$, temos que $v_{i}\left(d_{i}-1\right), v_{i}\left(d_{j}\right)>2 \delta_{i}$. Com isso, segue que $v_{i}\left(x-a_{i}\right)=v_{i}\left(a_{1} d_{1}+\ldots+a_{i}\left(d_{i}-1\right)+\ldots+a_{n} d_{n}\right)>\min _{1 \leq j \leq n}\left\{v_{i}\left(a_{j}\right)+2 \delta\right\} \geq-\delta_{i}+2 \delta_{i}=$ $\delta_{i} \geq \gamma_{i}$, como desejado. 


\section{CONSIDERAÇÕES FINAIS (ou Conclusão)}

Estudamos a topologia induzida por uma valorização em um corpo $K$. Além disso, descobrimos que a dependência dos anéis de valorização nada mais é do que induzir a mesma topologia em $K$.

\section{REFERÊNCIAS}

[1] ENGLER, A. J.; PRESTEL, A. Valued Field. Springer-Verlag, 2006. 